\title{
Evolution of Oxygen Deficiency Center on Fused Silica Surface Irradiated by Ultraviolet Laser and Posttreatment
}

\author{
Hai-Bing Lü,, ${ }^{1,2}$ Shi-Zhen Xu, ${ }^{1,3}$ Hai-Jun Wang, ${ }^{1}$ Xiao-Dong Yuan, \\ Chao Zhao, ${ }^{2}$ and Y. Q. Fu ${ }^{2}$ \\ ${ }^{1}$ Research Center of Laser Fusion, China Academy of Engineering Physics (CAEP), P.O. Box 919-988-5, Mianyang 621900, China \\ ${ }^{2}$ Thin Film Centre, Scottish Universities Physics Alliance (SUPA), University of the West of Scotland, Paisley PA1 2BE, UK \\ ${ }^{3}$ School of Physical Electronics, University of Electronic Science and Technology of China, Chengdu, Sichuan 610054, China \\ Correspondence should be addressed to Hai-Bing Lü; haibinglv@163.com and Y. Q. Fu; richard.fu@uws.ac.uk
}

Received 7 February 2014; Accepted 11 May 2014; Published 29 May 2014

Academic Editor: Haiyan Xiao

Copyright (C) 2014 Hai-Bing Lü et al. This is an open access article distributed under the Creative Commons Attribution License, which permits unrestricted use, distribution, and reproduction in any medium, provided the original work is properly cited.

\begin{abstract}
Evolution of oxygen deficiency centers (ODCs) on a fused silica surface irradiated using a $355 \mathrm{~nm}$ ultraviolet (UV) laser beam in both vacuum and atmospheric conditions was quantitatively studied using photoluminescence and X-ray photoelectron spectroscopy. When the fusedsilica surface was exposed to the UV laser in vacuum, the laser damage threshold was decreased whereas the concentration of the ODCs was increased. For the fuse silica operated under the high power lasers, creation of ODCs on their surface resulted from the UV laser irradiation, and this is more severe in a high vacuum. The laser fluence and/or laser intensity have significant effects on the increase of the ODCs concentration. The ODCs can be effectively repaired using postoxygen plasma treatment and UV laser irradiation in an excessive oxygen environment. Results also demonstrated that the "gain" and "loss" of oxygen at the silica surface is a reversible and dynamic process.
\end{abstract}

\section{Introduction}

Silica optics operated in vacuum generally show a poor stability when they are exposed to high-power laser systems. Some common damage of the silica optics includes (a) thermal damage when operated using the fundamental harmonic $(1 \omega)$ laser and (b) photoionization when operated using the third harmonic $(3 \omega)$ laser. It is generally accepted that the silica optics can be safely performed below their damage thresholds. However, surface damage easily occurs when the optics are operated in vacuum, and their damage thresholds are even lower than those measured at an atmospheric condition. The phenomenon is correlated with oxygen deficiency and has been widely investigated to elucidate the damage mechanisms [1-6].

Some luminescent defects in the fused silica are well known, for example, the oxygen deficiency centers (ODCs), nonbridging oxygen hole centers (NBOHCs), the peroxy radicals (PORs), and the singlet oxygen transition associated with interstitial neutral molecular oxygen $\left(\mathrm{O}_{2}\right)$, with their emission bands at $2.7 \mathrm{eV}(454 \mathrm{~nm}), 1.9 \mathrm{eV}(650 \mathrm{~nm}), 2.2 \mathrm{eV}$, and $1.2 \mathrm{eV}$, respectively [4-9].

A few researchers $[10,11]$ experimentally observed degradation in performance of the silica optics and found the oxygen deficiency centers (ODCs) on the surface of the silica optics using photoluminescence (PL) analysis. However, they did not provide the explanation of the detailed formation mechanisms of the ODCs in the fused silica optics. So far, evolution of the ODCs on the surface of the fused silica irradiated by UV laser has been rarely reported.

To reduce the ODCs on the surface of the fused silica, high temperature annealing of the fused silica for several hours in oxygen atmosphere has generally been used [12, 13]. Ion beam etching has also been used to remove the surface layer rich in defects in order to restore the damage threshold of the fused silica [6]. As an important component for high-power laser systems, the fused silica requires a high quality surface finish; thus, the heat treatment method at high temperature is obviously not preferred. In this paper, two methods have been applied to decrease the concentration of 


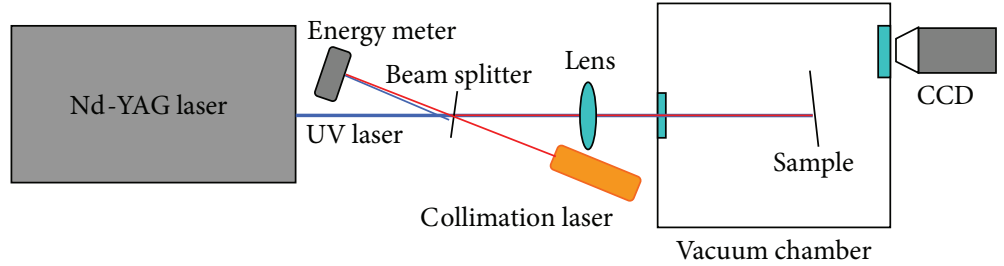

FIGURE 1: Arrangement of experimental system.

ODCs: (1) plasma treatment with low temperature oxygen plasma and (2) irradiation using a pulse UV laser in a pure oxygen atmosphere.

\section{Experimental}

The experimental system is schematically shown in Figure 1. Laser irradiation experiments were conducted using an NdYAG laser beam with a frequency of $355 \mathrm{~nm}$. The full width at half maximum (FWHM) of the laser pulse was $7.5 \mathrm{~ns}$, and the laser repetition rate was $10 \mathrm{~Hz}$.

The fluence of the laser beam was adjusted by changing the distance from sample surface to lens. Experimental samples of the fused silica (Schott Company) had a diameter of $35 \mathrm{~mm}$ and a thickness of $5 \mathrm{~mm}$. A laser beam was introduced into the chamber with a based vacuum of about $10^{-3} \mathrm{~Pa}$ achieved using a turbo pump and a mechanical roughing pump. The surface damage was monitored in situ using a CCD camera mounted on the chamber window. During laser irradiation, the fluence, duration time, or laser dose was changed. Some samples preexposed with laser beam in vacuum chamber were reirradiated using pulsed UV laser atmospheric conditions to clarify evolution processes of the ODCs.

The point defects of the ODCs induced by the UV laser preirradiation in the fused silica were characterized by measuring the photoluminescence (PL) spectra using a fluorescent spectrometer (F900, Edinburgh Instruments Ltd.). The PL intensity is corresponding to the concentration of the ODCs. Spectra of the Si 2p, O 1s, and C 1s were obtained using X-ray photoelectron spectroscopy (XPS, ESCALAB 250 system). The $\mathrm{X}$-ray source was a monochromatic $\mathrm{Mg} \mathrm{K}_{\alpha}$ beam with an energy of $1253.6 \mathrm{eV}$. The $\mathrm{Si}$ : $\mathrm{O}$ ratios on the surfaces of the silica optics under different irradiation conditions were obtained from analysis of the XPS spectra.

Two methods have been proposed in this study to repair preirradiated ODCs: (1) low temperature oxygen plasma treatment and (2) pulsed UV laser treatment. During plasma treatment, the chamber pressure was kept constant at $20 \mathrm{~Pa}$ and preformed using a Harrick plasma (PDC-001, 10W). UV laser treatment was conducted in atmospheric condition.

\section{Results and Discussion}

3.1. Effect of Laser Fluence. Figure 2 shows the emission bands of typical PL spectra, which are centered at $2.7 \mathrm{eV}$ (about $452 \mathrm{~nm}$ ) excited using a laser wavelength of $292 \mathrm{~nm}$. A peak centered at $\sim 452 \mathrm{~nm}$ is assigned to the ODCs. After

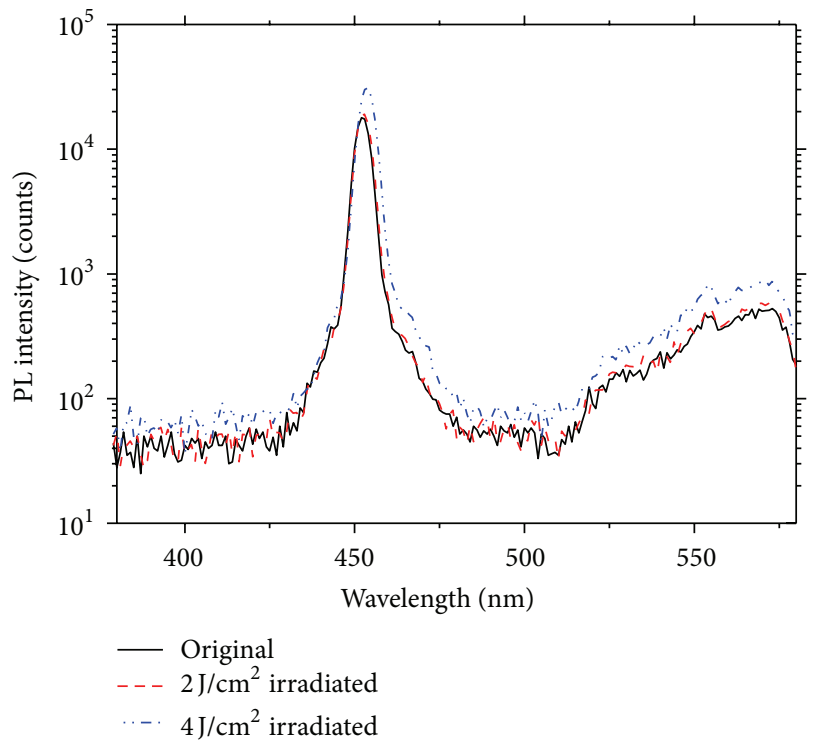

FIGURE 2: PL intensityof fused silica irradiated with different laser fluences and 30 laser pulses.

irradiation using UV laser at the fluences of $2 \mathrm{~J} / \mathrm{cm}^{2}$ and $4 \mathrm{~J} / \mathrm{cm}^{2}$ with 30 pulses, the PL peak intensity of the ODCs was increased from 1.78 to 1.91 and $3.11\left(\times 10^{4}\right.$ counts), respectively. The ODCs' concentration has been increased after pulsed UV laser irradiation in the vacuum $[4,5]$. Moreover, the $\mathrm{O}: \mathrm{Si}$ ratios on the surface of the fused silica obtained from the XPS analysis decreased after the laser irradiation. The O:Si ratio of the unirradiated (i.e., original fused silica sample) $2 \mathrm{~J} / \mathrm{cm}^{2}$ and $4 \mathrm{~J} / \mathrm{cm}^{2}$ irradiated samples with 30 laser pulses, obtained from XPS analysis, is $1.51,1.34$, and 1.32, respectively.

After the specimens were long-term (>20 hrs) irradiated using a continuous wave $(\mathrm{CW})$ laser with a wavelength of $355 \mathrm{~nm}$ and a power of $75 \mathrm{~mW}$ in vacuum, there is little increase of the PL peak intensity of the ODCs as shown in Figure 3. Clearly, the CW-UV laser irradiation in vacuum has little effect on concentration of the ODCs on surface of the fused silica.

The ODCs and peroxy radicals (PORs) are the primary defects on the surface of the fused silica after laser irradiation. The Frenkel-type mechanism has been used to explain this effect using the following $[14,15]$ :

$$
\equiv \mathrm{Si}-\mathrm{O}-\mathrm{Si} \equiv \longrightarrow \equiv \mathrm{Si}-\mathrm{Si} \equiv+\mathrm{O}_{2} \text { interstitial }
$$

(PORs and $\mathrm{O}_{2}$ ). 
TABLE 1: Effects of ODCs reduction on silica surface using two treatment methods.

\begin{tabular}{|c|c|c|c|c|}
\hline Treatment method & Original & $\begin{array}{l}\text { After UV irradiation } \\
\text { in vacuum }\end{array}$ & $\begin{array}{l}\text { Repaired with pulse UV } \\
\text { laser in excessive oxygen }\end{array}$ & $\begin{array}{c}\text { Repaired with oxygen } \\
\text { plasma }\end{array}$ \\
\hline $\begin{array}{l}\text { PL peak intensity } \\
\text { of ODCs/count }\end{array}$ & $4.55 \times 10^{4}$ & $6.25 \times 10^{4}$ & $3.51 \times 10^{4}$ & $3.51 \times 10^{4}$ \\
\hline
\end{tabular}

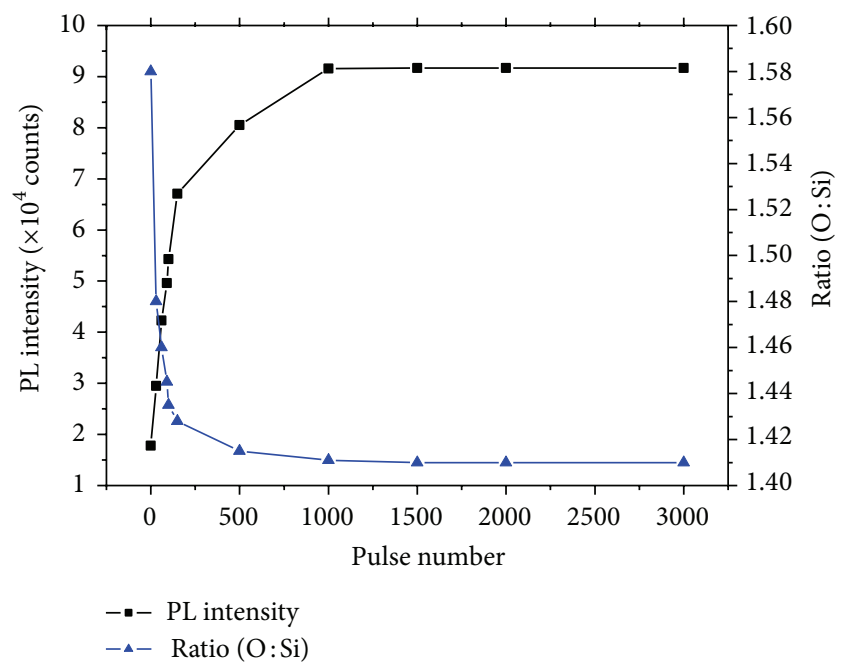

FIgURE 3: PL intensity and ratio $(\mathrm{O}: \mathrm{Si})$ of the silica optic samples as a function of pulse number (with a laser beam intensity of $3.1 \mathrm{~J} / \mathrm{cm}^{2}$ ).

The generated interstitial $\mathrm{O}_{2}$ gas may escape from the sample surface, resulting in oxygen vacancy and substoichiometric silica. Compared with laser irradiation in the atmospheric condition, the reactions (i.e., (1)) easily occur in vacuum. After the interstitial $\mathrm{O}_{2}$ was escaped from the sample surface, a substoichiometric $\mathrm{SiO}_{2}$, that is, $\mathrm{SiO}_{x}(0<x<$ 2 ), would form, which showed higher absorption levels and accounted for the continued degradation of the silica optics [4].

3.2. Effect of Laser Pulse Number. Figure 3 shows the effect of laser pulse number on the PL peak intensity of the ODCs. With the increase of pulse range from 1 to 1000, the PL intensity increased with the laser doses. However, the concentrations of the ODCs did not increase further when the number of laser pulses was above 1000. The O:Si ratio was obtained from the XPS data $[4,5]$ and the O:Si ratio decreases with increase of the laser irradiation doses. It shows that the formation rate of the ODCs (or the reaction shown in (1)) decreases, due to the increase of the generated defects and laser irradiation.

3.3. Reduction of ODCs. Figure 4 shows the changes of the PL peaks after laser irradiation in vacuum (red shift) and after plasma treatment, respectively, compared with those of the original sample. Table 1 summarizes the results of reduction of the ODCs using the two methods. From Table 1 and Figure 4, the PL intensity decreases after the plasma treatment, indicating that the concentration of the ODCs has

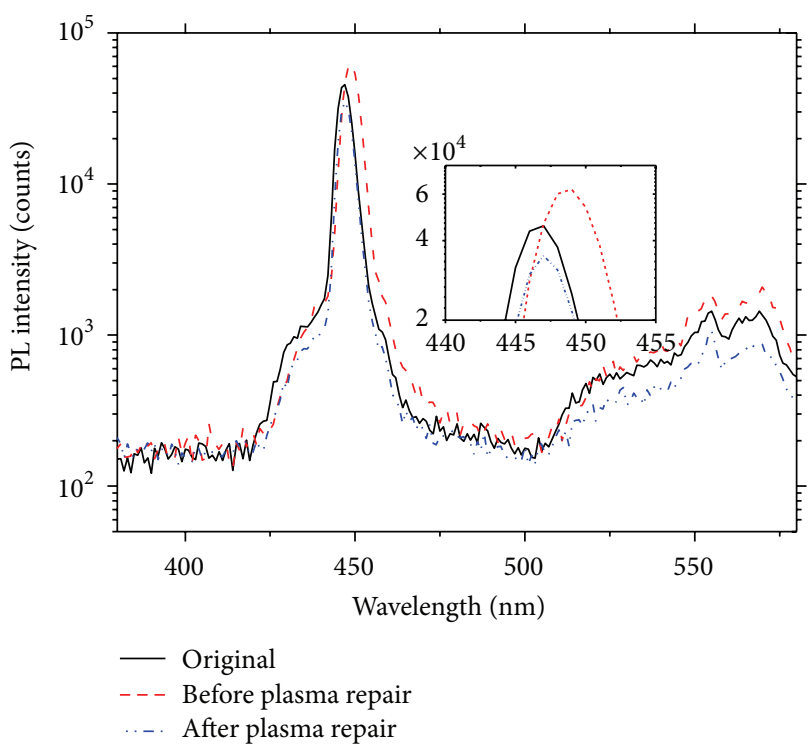

FIGURE 4: PL spectra of fused silica preirradiated in vacuum before and after oxygen plasma treatment.

been recovered. Clearly both the methods can be successfully used to repair the defects of the generation of ODCs.

For high quality $\mathrm{SiO}_{x}$ thin films, a PL peak was reported to have red shifts with decreasing oxygen concentration [16] and/or increasing the annealing temperature [17]. Such a red shift was also observed in this study using the silica, when its surface was exposed to nondamaging UV laser fluences in a vacuum environment (as shown in Figures 2 and 4). The close relationship between the luminescence signals and loss of oxygen on the surface of the silica can be evidenced from the observed back shift of the PL peak when the sample was illuminated by pulsed or CW-UV laser [18] in the presence of oxygen.

Above results showed that the "gain" and "loss" of oxygen elements on the silica surface is a reversible and dynamic process, which could reach a dynamic balance under a given laser irradiation condition. Oxygen plasma treatment and pulsed UV laser irradiation can decrease the ODCs and other defects to recover the performance of the fused silica. Using these methods, the lifetime of the fused silica optics used in high-power UV laser system can be prolonged when operated in an oxygen atmosphere.

\section{Conclusion}

For the fuse silica operated under the high-power lasers, creation of ODCs on their surface resulted from the UV laser 
irradiation, and this is more severe in a high vacuum. The laser fluence and/or laser intensity have significant effects on the increase of the ODCs concentration. The ODCs can be effectively repaired using postoxygen plasma treatment and UV laser irradiation in an excessive oxygen environment. Results also demonstrated that the "gain" and "loss" of oxygen at the silica surface is a reversible and dynamic process.

\section{Conflict of Interests}

The authors declare that there is no conflict of interests regarding the publication of this paper.

\section{Acknowledgments}

Financial support from Carnegie Trust Funding, Royal Society of Edinburgh, and Royal Academy of EngineeringResearch Exchange with China and India was acknowledged. This work is also supported by the Fundamental Research Funds for the Central Universities, China (Grant no. ZYGX2012J057).

\section{References}

[1] G. Pacchioni, L. Skuja, and D. L. Griscom, Eds., Defects in $\mathrm{SiO}_{2}$ and Related Dielectrics: Science and Technology, Kluwer Academic Publishers, Norwell, Mass, USA, 2000.

[2] R. A. B. Devine, J. P. Duraud, and E. Dooryhée, Eds., Structure and Imperfections in Amorphous and Crystalline Silicon Dioxide, John Wiley \& Sons, Chichester, UK, 2000.

[3] H. S. Nalwa, Ed., Silicon-Based Materials and Devices, Academic Press, San Diego, Calif, USA, 2001.

[4] S.-Z. Xu, H.-B. Lv, X.-D. Yuan et al., "Effects of vacuum on fused silica UV damage," Chinese Physics Letters, vol. 25, no. 1, pp. 223226, 2008.

[5] S. Xu, X. Zu, X. Jiang et al., "The damage mechanisms of fused silica irradiated by $355 \mathrm{~nm}$ laser in vacuum," Nuclear Instruments and Methods in Physics Research B, vol. 266, no. 1213, pp. 2936-2940, 2008.

[6] S. Xu, W. Zheng, X. Yuan, H. Lv, and X. Zu, "Recovery of fused silica surface damage resistance by ion beam etching," Nuclear Instruments and Methods in Physics Research B, vol. 266, no. 15, pp. 3370-3374, 2008.

[7] J. Fournier, J. Néauport, P. Grua et al., "Luminescence study of defects in silica glasses under near-UV excitation," Physics Procedia, vol. 8, pp. 39-43, 2010.

[8] R. Salh, "Defect related luminescence in silicon dioxide network: a review," in Crystalline Silicon-Properties and Uses, InTech, 2011.

[9] A. F. Zatsepin, E. A. Buntov, V. A. Pustovarov, and H.-J. Fitting, "Photoluminescence of Se-related oxygen deficient center in ion-implanted silica films," Journal of Luminescence, vol. 143, pp. 498-502, 2013.

[10] T. M. Stephen, B. van Zyl, and R. C. Amme, "Degradation of vacuum-exposed $\mathrm{SiO}_{2}$ laser windows," in 24th Annual Boulder Damage Symposium Proceedings-Laser-Induced Damage in Optical Materials, vol. 1848 of Proceedings of SPIE, pp. 106-109, 1992.

[11] A. N. Trukhin, K. M. Golant, Y. Maksimov, M. Kink, and R. Kink, "Recombination luminescence of oxygen-deficient centers in silica," Journal of Non-Crystalline Solids, vol. 354, no. 2-9, pp. 244-248, 2008.

[12] N. Shen, M. J. Matthews, S. Elhadj, P. E. Miller, A. J. Nelson, and J. Hamilton, "Correlating optical damage threshold with intrinsic defect populations in fused silica as a function of heat treatment temperature," Journal of Physics D: Applied Physics, vol. 46, no. 16, Article ID 165305, 2013.

[13] R. Salh, "Defect related luminescence in silicon dioxide network: a review," in Crystalline Silicon-Properties and Uses, InTech, 2011.

[14] T. E. Tsai and D. L. Griscom, "Experimental evidence for excitonic mechanism of defect generation in high-purity silica," Physical Review Letters, vol. 67, no. 18, pp. 2517-2520, 1991.

[15] H. Hosono, H. Kawazoe, and N. Matsunami, "Experimental evidence for frenkel defect formation in amorphous $\mathrm{SiO}_{2}$ by electronic excitation," Physical Review Letters, vol. 80, no. 2, pp. 317-320, 1998

[16] T. Mohanty, N. C. Mishra, S. V. Bhat, P. K. Basu, and D. Kanjilal, "Dense electronic excitation induced defects in fused silica," Journal of Physics D, vol. 36, no. 24, pp. 3151-3155, 2003.

[17] S. Hayashi, T. Nagareda, Y. Kanzawa, and K. Yamamoto, "Photoluminescence of Si-rich $\mathrm{SiO}_{2}$ films: Si clusters as luminescent centers," Japanese Journal of Applied Physics, vol. 32, no. 9, pp. 3840-3845, 1993.

[18] S. G. Demos, A. Burnham, P. Wegner et al., "Surface defect generation in optical materials under high fluence laser irradiation in vacuum," Electronics Letters, vol. 36, no. 6, pp. 566-567, 2000. 

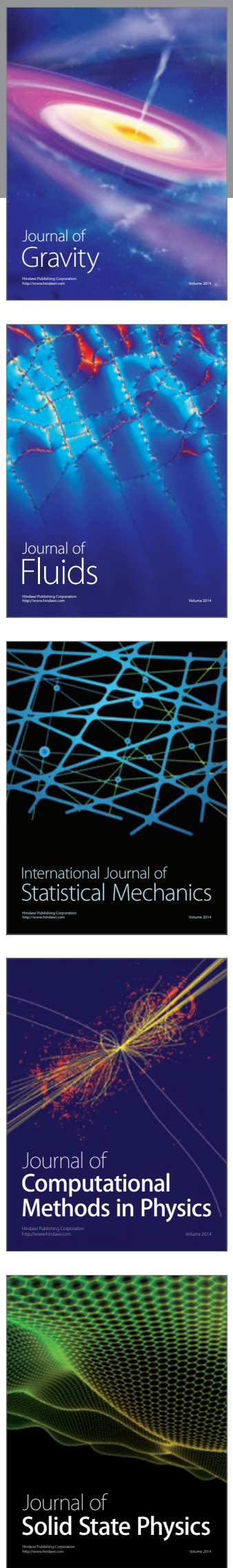

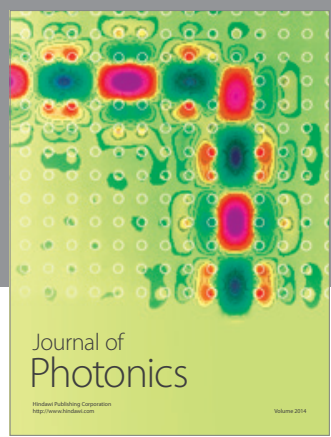

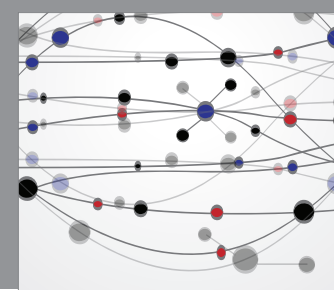

The Scientific World Journal

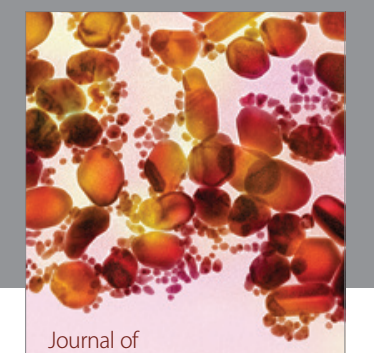

Soft Matter
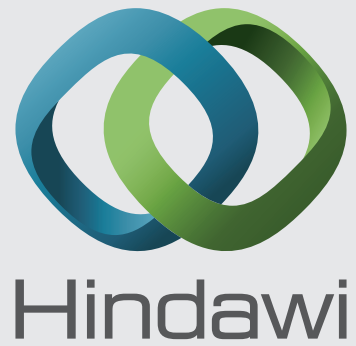

Submit your manuscripts at

http://www.hindawi.com
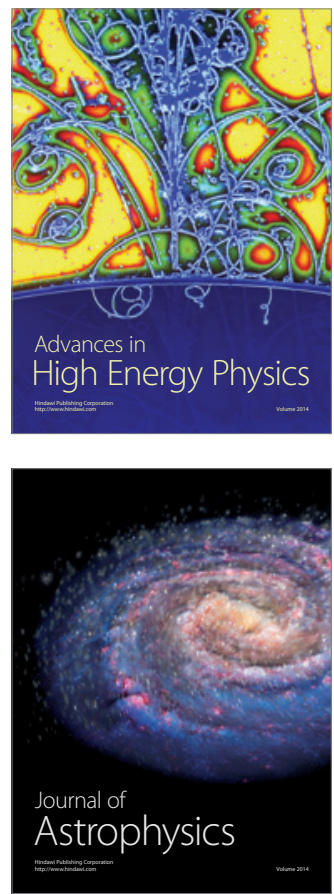
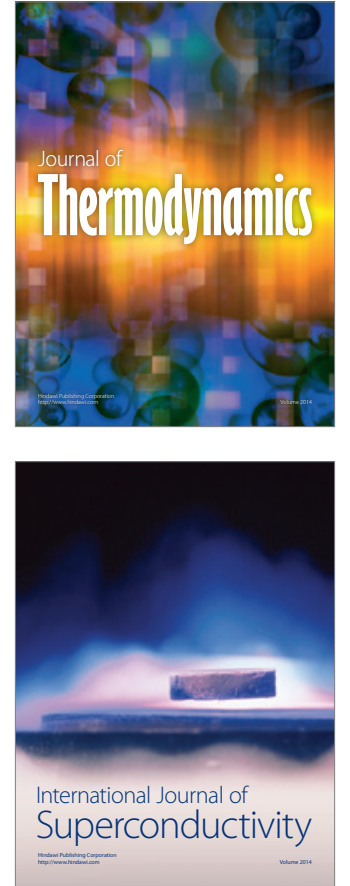
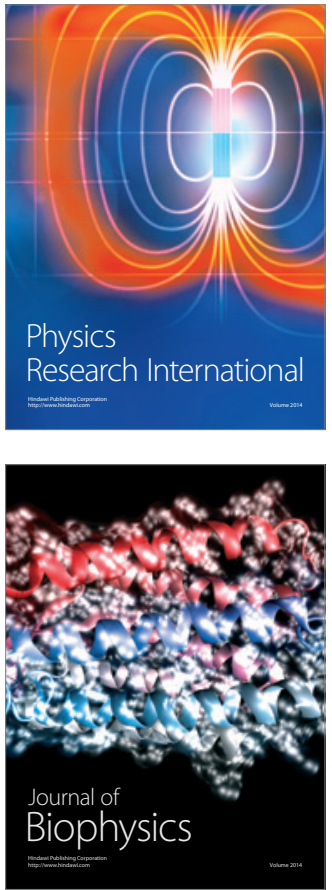
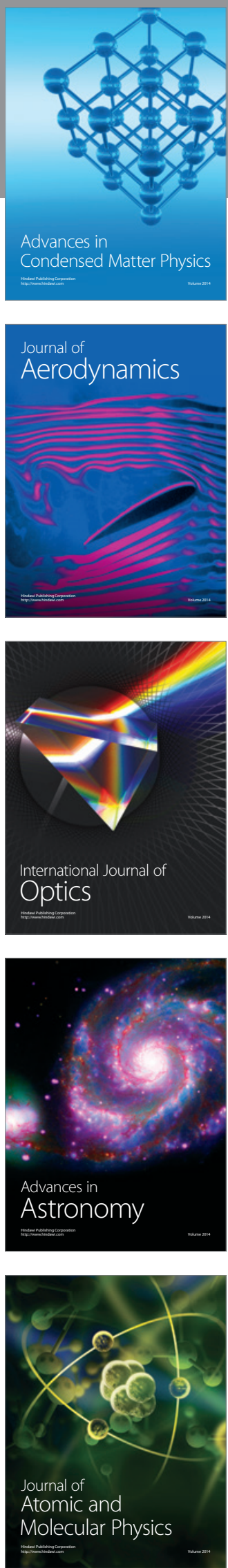